\title{
Study of emulsion explosive quality assessment system based on soft measurement and multilevel fuzzy evaluation
}

\author{
Yuesheng Wang*, and Chenqi Huang \\ School of Hangzhou Dianzi University, Hangzhou, China
}

\begin{abstract}
Aiming at the inaccuracy and blindness of the traditional detection and assessment methods for the quality of emulsion explosives, a method for evaluating the quality of emulsion explosives based on soft measurement and multilevel fuzzy evaluation is proposed. The soft-sensing model of BP neural network can predict the online unmeasured performance indicators of detonation velocity and detonation online. The multilevel fuzzy evaluation method establishes the reliable multilevel fuzzy evaluation system based on the key parameters of the production process and expert experience. Experiments show that this soft-sensing model have made the convergence quickly and the accuracy highly, and can accurately predicts the detonation velocity and brisance of emulsion explosives online. In the last part, the design of quality assessment system can provide a new idea for solving the point of quality blindness detection and assessment.
\end{abstract}

\section{Introduction}

As a new type of industrial explosive, while the output of emulsion explosives is increasing day by day, the number of substandard products is also increasing.Enhancing the quality management of emulsion explosive products is the key for companies to survive in the fierce competition [1]. At present, there are two methods for quality inspection of emulsion explosives: the sampling inspection method and process parameter detection method. The facts show that although the former can accurately detect quality, the sampling period is too long; while the latter can detect process parameters online, it cannot accurately test quality. And there is blindness that people subjectively evaluate the quality of explosives. Therefore, it is significant to find a scientific and effective method for the detection and evaluation of the quality of emulsion explosives.

\section{The soft measurement of emulsion explosives performance indicators}

\footnotetext{
* Corresponding author: wys@hdu.edu.cn
} 


\subsection{Introduction of the soft measurement}

Soft measurement technology is based on the easy detection of process variables,making use of mathematical relationships between easy-to-measure process variables and process variables, through a variety of mathematics calculate and estimate the measurement of the process variable to be measured [2]. As an emerging industrial control technology and comprehensive automation technology, soft measurement technology is not only economical and reliable, but also has a rapid dynamic response. It has broad application prospects for development [3].

\subsection{Soft measurement model of detonation velocity and brisance}

The quality of emulsion explosives is mainly reflected in the detonation performance,and the detonation performance is determined by the detonation velocity and brisance . In order to achieve the purpose of detection and evaluation of the emulsion explosives quality online. This article takes the detonation velocity and brisance as the output of the soft measurement. The flow chart of the emulsion explosives production is shown in figure 1.

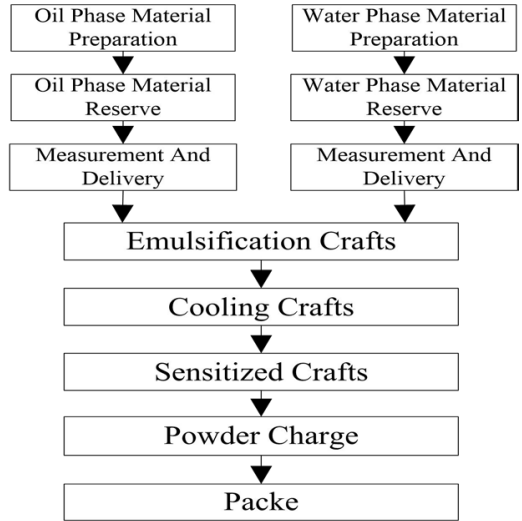

Fig. 1. The flow chart of the emulsion explosives production.

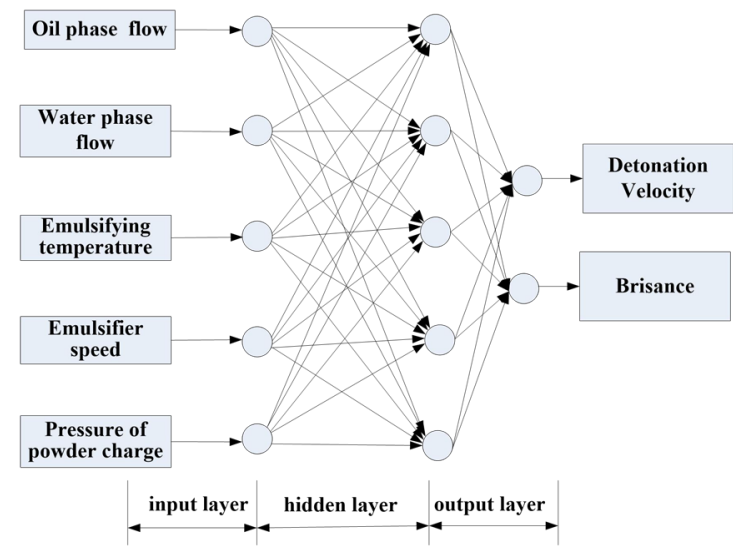

Fig. 2. The structure of BP neural network.

\subsubsection{Selection of the input variables}

After analysing the production process of emulsion explosives, the main parameters affecting the detonation velocity and brisance are obtained:the oil phase flow, the water phase flow, the emulsification temperature,the emulsifier speed and the pressure of powder charge. Therefore, these five parameters are as input for the soft measurement model.

Due to the unstable start-up of the emulsion explosive production, this paper selects 200 sets of data after normal production for a period of time. 150 sets of data are used as training samples, and another 50 sets of data are used as test samples, each set of data contains each of the above parameters. For data transformation, the data normalization method can eliminate the influence of different dimensions and different orders of magnitude of measurement data.

\subsubsection{Establishment of soft measurement model}


Since the three-layer BP neural network can solve the nonlinear problem [4], the number of hidden layers is chosen to be 1.The determination of the number of nodes in the hidden layer is a complex problem. If the number of nodes selected is too few, the generalization ability and fault tolerance of the network will be poor, but too many middle layer nodes will be over-fitted. This article bases on the following empirical equation (1):

$$
s=\sqrt{m+n}+a
$$

As the equation (1) shows , $m$ represent the number of input nodes, $m=5 ; n$ is the number of output nodes, $n=2 ; a$ is constant of $1 \sim 10$.First determining the approximate range of the number of nodes in the middle layer, and then through the simulation test to obtain the number of neurons as the last best network performance prediction of the number of neurons network [5], in this case $a=5$. The BP neural network structure is shown in figure 2 .

The common BP network is not suitable for practical use. By consulting the literature, the Levenberg-Marquardt algorithm is suitable for medium-sized BP neural network, and the convergence speed is fast, also the precision is high. Therefore, the BP neural network improved by the LM algorithm is used in this model. The hidden layer transfer function uses the tan-Sigmoid function, the output layer uses the purelin function, setting the target error to 0.01 .

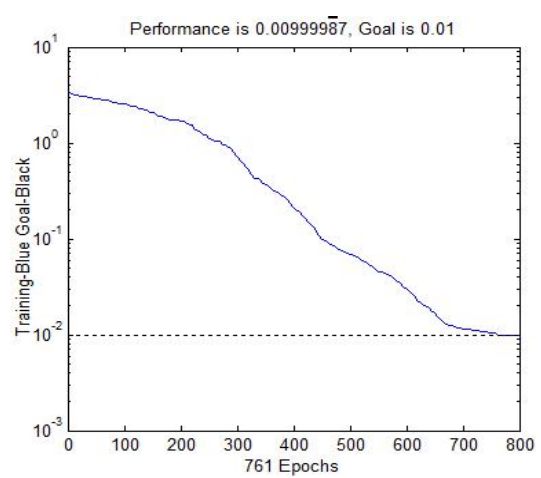

Fig. 3. BP training error performance.

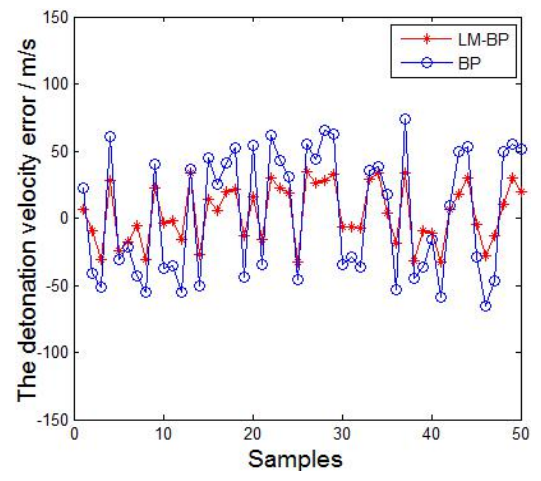

Fig. 5.The detonation velocity error.

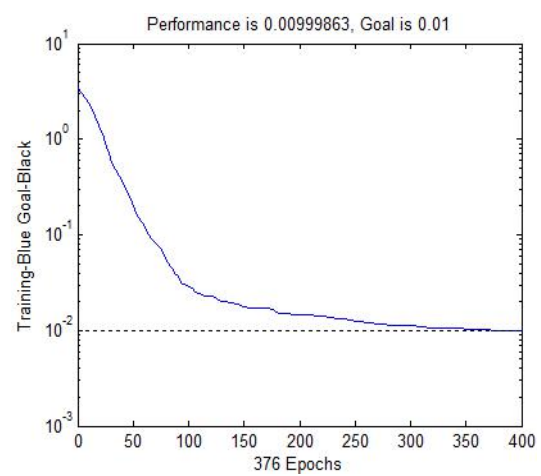

Fig. 4. LM-BP training error performance

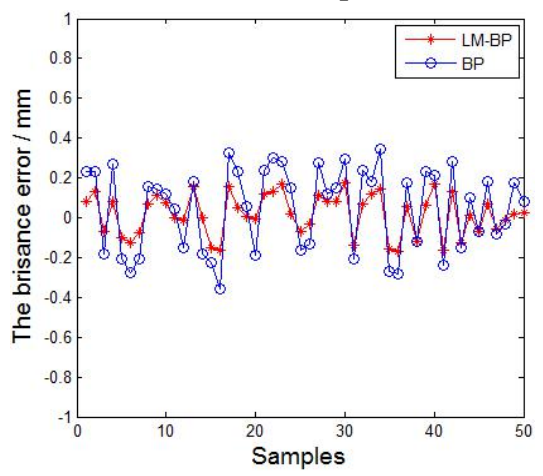

Fig. 6. The brisance error.

As shown in figure 3 and figure 4, we can see that the LM-BP network training to 376 steps, and converges fastly.

From figure 4 and figure 5, we can see that the LM-BP has a smaller predicted output error. The soft-sensing model established by the improved BP neural network can accurately predict the detonation velocity and brisance of emulsion explosives. 


\section{Emulsion Explosives Quality Evaluation System}

\subsection{Establishment of Emulsion Explosives Quality Evaluation System}

\subsubsection{Multilevel fuzzy evaluation system}

There are many fuzzy evaluation concepts that people can not use a strict boundary to distinguish them in the actual evaluation. The multilevel fuzzy evaluation is based on fuzzy mathematics, and quantifies fuzzy factors of the unclear boundaries. In other words, this method can comprehensively assess the membership degree of objects from multiple factors. The steps of this method are as follows [6]:

(1) Set $U$ for establishing fuzzy comprehensive evaluation factors.

(2) Set V for the comment, and the score of each rank in the comment is converted to $\mathrm{V}^{\prime}$.

(3) Determine the fuzzy relation matrix $R$ and the weight $A$.

(4) Synthesis quality assessment result vector W, as is shown in equation (2).

$$
W=A \cdot R
$$

(5) The evaluation result is scored to $P$, as is shown in equation (3).

$$
P=V^{\prime} \cdot W
$$

\subsubsection{Determination of important parameters}

Due to the many and complex factors affecting the quality of emulsion explosives, this article analyzes the production process and combines expert experience to determine the set of factors for evaluating the quality of emulsion explosives. As shown in table 1, the "detonation Velocity" and "Brisance" obtained from the soft measurement are added to the "Process Parameter" index. The "Process Parameters", the "Environmental Parameters", the "Materials" and the "Equipment" are set to 4 first-level assessment indicators, and set 11 indicators as secondary indicators.According to the assessment target of the quality, this article sets $V=\{$ Excellent, Good, Qualified, Unqualified $\}$, and set $V^{\prime}=\{100,80,60,40\}$, and then combine with the evaluation factor, determine the membership degree of each index corresponding to the four levels of the comment, so as to determine the second-level index fuzzy relationship matrix belonging to each first-level index:

$$
R=\left(r_{i j m \times 4}\right)=\left[\begin{array}{cccc}
r_{11} & r_{12} & r_{13} & r_{14} \\
r_{21} & r_{22} & r_{33} & r_{44} \\
\cdots & \ldots & \ldots & \ldots \\
r_{m 1} & r_{m 2} & r_{m 3} & r_{m 4}
\end{array}\right] \quad i=1,2, \ldots m ; j=1,2,3,4
$$

The emulsion explosive quality evaluation system contains quantitative and qualitative index. For the Process parameters and Environmental parameters, the membership function belonging to the lowest level index is constructed to determine the degree of the secondary index; for the Materials and the Equipment, the degree is determined by an expert assessment method.

As shown in table 1, this paper uses the Analytic Hierarchy Process (AHP) to determine the weight, firstly analyzes the relationship among the indicators within the same level, calculates the weight through the judgment matrix, and determines the index weight for each level after the consistency test [7]. 
Table 1. All levels of the assessment factors and the weight.

\begin{tabular}{|c|c|c|}
\hline $\begin{array}{l}\text { Comprehensive } \\
\text { Indicators / } \\
\text { Weight }\end{array}$ & $\begin{array}{l}\text { First-Level } \\
\text { Indicators/Weight }\end{array}$ & Second-Level Indicators / Weight \\
\hline \multirow{11}{*}{$\begin{array}{l}\text { Emulsion } \\
\text { explosive } \\
\text { quality(A) }\end{array}$} & \multirow{7}{*}{$\begin{array}{l}\text { Process parameters } \\
\text { (B1)/0.9202 }\end{array}$} & Emulsification time/(0.0894) \\
\hline & & Temperature of the oil phase $/(0.0456)$ \\
\hline & & Temperature of the water phase $/(0.0456)$ \\
\hline & & $\begin{array}{l}\text { Temperature of cooling stage matrix } \\
/(0.0458)\end{array}$ \\
\hline & & Sensitization time $/(0.0958)$ \\
\hline & & Detonation velocity /(0.3389) \\
\hline & & Brisance /(0.3389) \\
\hline & \multirow{2}{*}{ Environmental(B2)/0.1245 } & Atmospheric pressure/(0.3333) \\
\hline & & Environmental temperature/(0.6667) \\
\hline & Materials(B3)/0.3182 & Quality standards/(1) \\
\hline & Equipment(B4)/0.1911 & Unaged equipment/(1) \\
\hline
\end{tabular}

\subsection{Design of Emulsion Explosives Quality Evaluation System}

As is shown in figure 6, this part is design on the computer.The software module includes the data acquisition, the data display, the soft measurement and the explosive quality assessment. This system is developed on the basis of the original system, acquire the realtime data from the original system's King-view software. After evaluating the explosive quality, the evaluation results are displayed together with real-time data on the software interface.

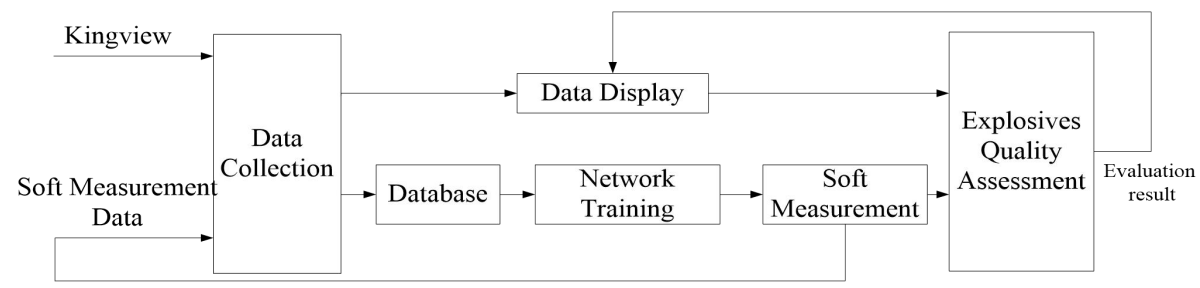

Fig. 6. The overall structure of emulsion explosive quality evaluation system.

\section{Conclusion}

This paper proposes a quality assessment system for emulsion explosives based on soft measurement and multilevel fuzzy evaluation methods. A soft measurement model is built using BP neural network to make the explosives performance parameters measurable online.The simulation results show that this model can accurately predict detonation velocity and brisance online.The last part designs the emulsion explosive quality evaluation system based on the soft measurement and multilevel fuzzy evaluation method, and provides a new idea for establishing a scientific and effective explosive quality evaluation system. 


\section{References}

1. Suli Men, Jun Yang, Weixin Huang, Talking about the Quality Management of Civil Explosive Enterprises[J]. Guangdong Chemical Industry. 16(42):151-52 (2015)

2. Jinshou $\mathrm{Yu}$, The application of soft sensing technique in petrochemical industry[J]. Petroleum and chemical industry.29(3):221-26 (2000)

3. Xiuliang Li, Research and Application of Soft Measurement Modeling Method[D]. Zhejiang: Zhejiang University, 1-2 (2009)

4. Yongji Wang, Jian Tu, Network control[M]. Beijing:Machinery Industry Press, 34-44 (1998)

5. Wusheng $\mathrm{Hu}$, The theory of neural network and its application in Engineering[M]. Beijing: Publishing House of Surveying and mapping, 63-83 (2005)

6. Zhao Zilan, Energy-saving Potential Comprehensive Evaluation of Distribution Network Based on Multilevel Fuzzy Appraisal[C]. Beijin: North China Electric Power University, 25-30 (2014)

7. Zhao Kai, Li Weiyao, Fault Analysis of Machine Tool Based on Fuzzy Analytic Hierarchy Process[J]. Shanghai:Microcomputer Applications, 72-75 (2015) 